\title{
The Beta Transmuted Weibull Distribution
}

\author{
Manisha Pal \\ Calcutta University
}

\author{
Montip Tiensuwan \\ Mahidol University
}

\begin{abstract}
The paper introduces a beta transmuted Weibull distribution, which contains a number of distributions as special cases. The properties of the distribution are discussed and explicit expressions are derived for the mean deviations, Bonferroni and Lorenz curves, and reliability. The distribution and moments of order statistics are also studied. Estimation of the model parameters by the method of maximum likelihood is discussed. The log beta transmuted Weibull model is introduced to analyze censored data. Finally, the usefulness of the new distribution in analyzing positive data is illustrated.
\end{abstract}

Keywords: reliability function, moment generating function, mean deviation, Bonferroni and Lorenz curve, reliability and entropies, maximum likelihood estimation.

\section{Introduction}

The Weibull distribution is a very popular life time probability distribution that has been extensively used for modeling in reliability, engineering and biological studies. Generalizations of the distribution have been suggested by many authors. Sarhan and Zaindin (2009) studied the modified Weibull distribution, Mudholkar and Srivastava (1993) introduced the exponentiated Weibull distribution and Pal, Masoom Ali, and Woo (2006) investigated many of its properties. Elbatal (2011) studied the exponentiated modified Weibull distribution.

A class of generalized distributions $F(x)$ has been receiving considerable attention over the last few years, in particular, after the studies by Eugene, Lee, and Famoye (2002) and Jones (2004). If $G$ denotes the baseline cumulative distribution function (cdf) of a random variable, then the beta- $G$ distribution is defined as

$$
F(x)=I_{G(x)}(a, b)=\frac{1}{B(a, b)} \int_{0}^{G(x)} w^{a-1}(1-w)^{b-1} d w, \quad 0<a, 0<b .
$$

Here, $I_{y}(a, b)=B_{y}(a, b) / B(a, b)$ is the incomplete beta function ratio, $B_{y}(a, b)=\int_{0}^{y} w^{a-1}(1-$ $w)^{b-1} d w$ is the incomplete beta function and $B(a, b)=\Gamma(a) \Gamma(b) / \Gamma(a+b)$ is the beta function, where $\Gamma(\cdot)$ is the gamma function. The probability density function (pdf) of the above distribution has the form

$$
f(x)=\frac{1}{B(a, b)} G(x)^{a-1}\{1-G(x)\}^{b-1} g(x), \quad x>0 .
$$

Based on the above generalization, Lee, Famoye, and Olumolade (2007) introduced the beta-Weibull distribution. Thereafter, Silva, Ortega, and Cordeiro (2010) investigated the beta modified Weibull 
distribution, and Cordeira, Gomes, da-Silva, and Ortega (2013) made a detailed study of the betaexponentiated Weibull distribution.

Recently, Aryall and Tsokos (2011) introduced another generalization of the Weibull distribution, which they called the transmuted Weibull distribution. A random variable $T$ is said to have transmuted Weibull probability distribution with parameters $\alpha, \beta>0$ and $|\lambda| \leq 1$, if it has the pdf given by

$$
g_{T W}(x)=\alpha \beta x^{\beta-1} \exp \left(-\alpha x^{\beta}\right)\left(1-\lambda+2 \lambda \exp \left(-\alpha x^{\beta}\right)\right), \quad x>0,
$$

where $\alpha$ and $\beta$ are the shape parameters representing the different patterns of the transmuted Weibull distribution and are positive, and $\lambda$ is the transmuted parameter.

The cdf of the transmuted Weibull distribution is obtained as

$$
G_{T W}(x)=\left(1-\exp \left(-\alpha x^{\beta}\right)\right)\left(1+\lambda \exp \left(-\alpha x^{\beta}\right)\right), \quad x>0 .
$$

In this paper, we introduce and study several mathematical properties of a new distribution, referred to as a beta transmuted Weibull (BTW) distribution. The distribution has two extra shape parameters which provide greater flexibility in modelling observed positive data. The paper is organized as follows. In Section 2, we introduce the distribution. In Sections 3, we obtain expansions of the cdf and pdf of the distribution using power series. Quantile function and mean deviation are derived in Sections 4 and 5. Order statistics and their moments are discussed in Sections 6 and 7. In Section 8, the stress-strength reliability is obtained. Estimation of parameters by the maximum likelihood method is discussed in Section 9. In Section 10, log beta transmuted Weibull regression model is investigated. In Section 12, the distribution is used for analyzing real life data. Finally, in Section 13, we make some concluding remarks on our study.

\section{The beta transmuted Weibull distribution}

The five-parameter BTW distribution is obtained by taking $G(x)$ in (1) to be the cdf of a transmuted Weibull distribution given by (4). The BTW cdf then becomes

$$
\begin{aligned}
F(x) & =I_{\left(1-\exp \left(-\alpha x^{\beta}\right)\right)\left(1+\lambda \exp \left(-\alpha x^{\beta}\right)\right)}(a, b) \\
& =\frac{1}{B(a, b)} \int_{0}^{\left(1-\exp \left(-\alpha x^{\beta}\right)\right)\left(1+\lambda \exp \left(-\alpha x^{\beta}\right)\right)} w^{a-1}(1-w)^{b-1} d w, \quad x>0,
\end{aligned}
$$

where $\alpha>0, \beta>0,|\lambda| \leq 1$, and $a>0, b>0$.

The cdf can be expressed in a closed form using the hypergeometric function (see Cordeiro and Nadarajah 2011) as follows:

$$
\begin{aligned}
F(x)= & \frac{\left\{\left(1-\exp \left(-\alpha x^{\beta}\right)\right)\left(1+\lambda \exp \left(-\alpha x^{\beta}\right)\right)\right\}^{a}}{a B(a, b)} \\
& { }_{2} F_{1}\left(a, 1-b ; a+1 ;\left(1-\exp \left(-\alpha x^{\beta}\right)\right)\left(1+\lambda \exp \left(-\alpha x^{\beta}\right)\right)\right),
\end{aligned}
$$

where

$$
{ }_{2} F_{1}(c, d ; e ; z)=\sum_{k=0}^{\infty} \frac{(c)_{k}(d)_{k}}{k !(e)_{k}} z^{k}
$$

is the Gaussian hypergeometric function with $(c)_{k}$ defined as

$$
\begin{aligned}
& (c)_{k}=c(c+1)(c+2) \cdots(c+k-1), \quad k=1,2, \ldots \\
& (c)_{0}=1 .
\end{aligned}
$$


The pdf $f(x)$ and the hazard rate function $h(x)$ are obtained as

$$
\begin{aligned}
f(x)= & \frac{1}{B(a, b)} \alpha \beta t^{\beta-1} \exp \left(-\alpha t^{\beta}\right)\left(1-\lambda+2 \lambda \exp \left(-\alpha t^{\beta}\right)\right) \\
& \left(1-\exp \left(-\alpha x^{\beta}\right)\right)^{a-1}\left(1+\lambda \exp \left(-\alpha x^{\beta}\right)\right)^{a-1} \\
& \left\{1-\left(1-\exp \left(-\alpha x^{\beta}\right)\right)\left(1+\lambda \exp \left(-\alpha x^{\beta}\right)\right)\right\}^{b-1}, \quad t>0, \\
h(x)= & \frac{\alpha \beta x^{\beta-1} \exp \left(-\alpha x^{\beta}\right)\left(1-\lambda+2 \lambda \exp \left(-\alpha x^{\beta}\right)\right)}{B(a, b) I_{1-\left(1-\exp \left(-\alpha x^{\beta}\right)\right)\left(1+\lambda \exp \left(-\alpha x^{\beta}\right)\right)}(b, a)} \\
& \left(1-\exp \left(-\alpha x^{\beta}\right)\right)^{a-1}\left(1+\lambda \exp \left(-\alpha x^{\beta}\right)\right)^{a-1} \\
& \left\{1-\left(1-\exp \left(-\alpha x^{\beta}\right)\right)\left(1+\lambda \exp \left(-\alpha x^{\beta}\right)\right)\right\}^{b-1}, \quad t>0 .
\end{aligned}
$$

Plots of the pdf (6) and the hazard rate function (7) for some values of $\alpha, \beta, \lambda, a$ and $b$ are given in Figures 1 and 2, respectively. The BTW failure rate function can be monotonically decreasing or increasing and upside-down bathtub depending on the values of its parameters.

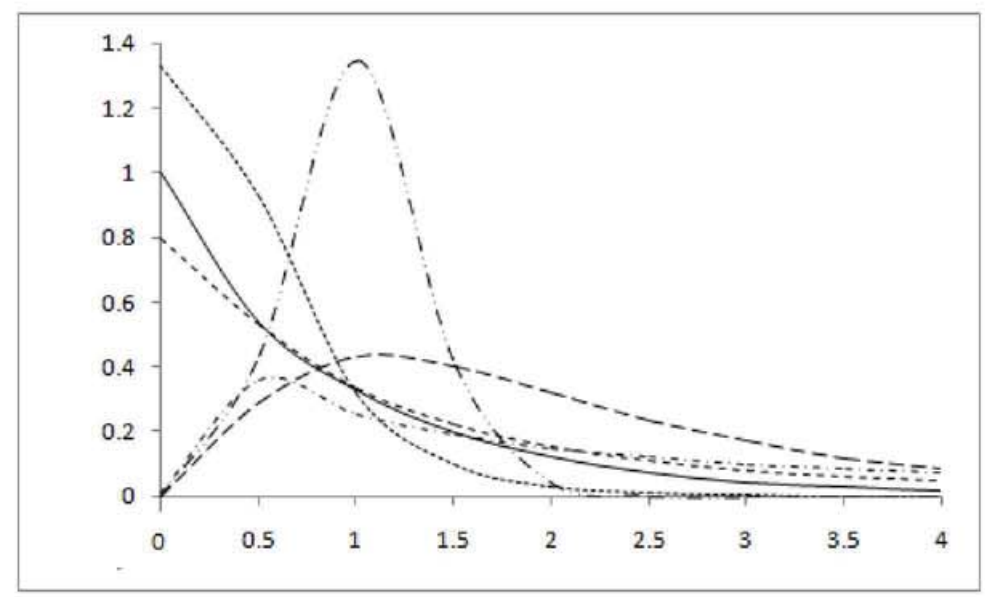

Figure 1: Pdf of beta transmuted Weibull distribution for $\alpha=1$ and (i) $\beta=1, \lambda=0, a=1, b=1$, (ii) $\beta=1, \lambda=0.2, a=3, b=0.75$, (iii) $\beta=0.5, \lambda=0.5, a=3, b=0.75$, (iv) $\beta=2, \lambda=1$, $a=3, b=0.75$, (v) $\beta=1.5, \lambda=-1, a=3, b=0.75$, (vi) $\beta=1.3, \lambda=0.7, a=0.8, b=1.2$.

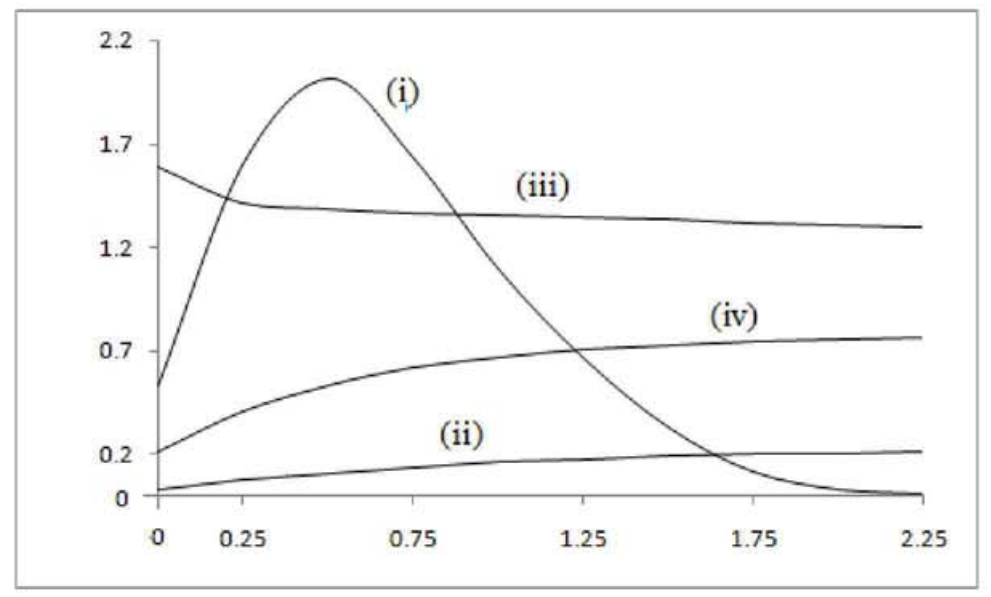

Figure 2: Hazard rate function of beta transmuted Weibull distribution for $\alpha=1$ and (i) $\beta=3$, $\lambda=0.5, a=1, b=2$, (ii) $\beta=0.75, \lambda=-1, a=2, b=0.75$, (iii) $\beta=0.75, \lambda=1, a=3, b=2$, (iv) $\beta=0.75, \lambda=-0.5, a=3, b=2$.

The following distributions are obtained from the BTW distribution by proper choice of its parameters: 


\begin{tabular}{ll}
\hline Parameters & Distribution \\
\hline$b=1$ & exponentiated transmuted Weibull \\
$a=b=1$ & transmuted Weibull \\
$\lambda=0$ & beta Weibull \\
$\lambda=0, b=1$ & exponentiated Weibull \\
$\lambda=0, a=b=1$ & Weibull \\
\hline$\beta=1$ & beta transmuted exponential \\
$\beta=1, a=b=1$ & transmuted exponential \\
$\beta=1, \lambda=0$ & beta exponential \\
$\beta=1, \lambda=0, b=1$ & exponentiated exponential \\
$\beta=1, \lambda=0, a=b=1$ & exponential \\
\hline
\end{tabular}

\section{Expansions for the CDF and PDF}

Here we express $F(x)$ and $f(x)$ in terms of infinite (or finite) weighted sums of cdf's and pdf's of Weibull distributions, respectively.

We note that for $b>0$ real non-integer, we can replace $(1-w)^{b-1}$ under the integral in (1) by the power series expansion of binomials and integrate to obtain

$$
\frac{1}{B(a, b)} \int_{0}^{G(x)} w^{a-1}(1-w)^{b-1} d w=\sum_{j=0}^{\infty}(-1)^{j}\left(\begin{array}{c}
b-1 \\
j
\end{array}\right) \frac{G(x)^{a+j}}{a+j}
$$

where the binomial term

$$
\left(\begin{array}{c}
b-1 \\
j
\end{array}\right)=\frac{\Gamma(b)}{\Gamma(b-j) j !}
$$

is defined for any real $b$. Then, from (5) we get

$$
F(x)=\sum_{j=0}^{\infty}(-1)^{j} \frac{\Gamma(b)}{\Gamma(b-j) j !} \frac{\left\{\left(1-\exp \left(-\alpha x^{\beta}\right)\right)\left(1+\lambda \exp \left(-\alpha x^{\beta}\right)\right)\right\}^{a+j}}{B(a, b)(a+j)}, \quad x>0 .
$$

Using the binomial expansion another two times we have for $x>0$

$$
\begin{aligned}
F(x) & =\sum_{j, k, l=0}^{\infty}(-1)^{j+k}\left(\begin{array}{c}
b-1 \\
j
\end{array}\right)\left(\begin{array}{c}
a+j \\
k
\end{array}\right)\left(\begin{array}{c}
a+j \\
l
\end{array}\right) \lambda^{l} \frac{\left.\exp \left(-\alpha(k+l) x^{\beta}\right)\right)}{B(a, b)(a+j)} \\
& =\sum_{j, k, l=0}^{\infty}(-1)^{j+k}\left(\begin{array}{c}
b-1 \\
j
\end{array}\right)\left(\begin{array}{c}
a+j \\
k
\end{array}\right)\left(\begin{array}{c}
a+j \\
l
\end{array}\right) \frac{\lambda^{l}\left\{1-G_{1}(x ; \alpha(k+l), \beta)\right\}}{B(a, b)(a+j)},
\end{aligned}
$$

where $G_{1}(\alpha(k+l), \beta)$ is the Weibull cdf with scale $\alpha(k+l)$ and shape $\beta$.

Differentiating (9) with respect to $x$ gives a useful expansion of $f(x)$ as

$$
f(x)=\sum_{k, l=0}^{\infty} w_{k l} g(x ; \alpha(k+l), \beta), \quad x>0,
$$

where

$$
w_{k l}=\sum_{j=0}^{\infty}(-1)^{j+k+1}\left(\begin{array}{c}
b-1 \\
j
\end{array}\right)\left(\begin{array}{c}
a+j \\
k
\end{array}\right)\left(\begin{array}{c}
a+j \\
l
\end{array}\right) \frac{\lambda^{l}}{B(a, b)(a+j)}
$$

and $g(x ; \alpha(k+l), \beta)$ is the Weibull pdf with scale $\alpha(k+l)$ and shape $\beta$. If $b>0$ is an integer, the index $j$ in the sum stops at $b-1$, and if $a$ is an integer, then the indices $k$ and $l$ in the sum stop at $a+j$.

The moments and the moment generating function of the BTW distribution can be easily expressed as functions of those quantities for Weibull distributions by using expression (10) of its pdf. 
If $X$ has a Weibull distribution with scale $\theta$ and shape $\delta$, we have

$$
\begin{aligned}
\mathrm{E}\left(X^{r}\right) & =\frac{1}{\theta^{r / \delta}} \Gamma(1+r / \delta), \\
M_{X}(t) & =\sum_{r=0}^{\infty} \frac{t^{r} \theta^{-r / \delta}}{r !} \Gamma(1+r / \delta), \quad \delta \geq 1 .
\end{aligned}
$$

Hence, for $X \sim$ BTW with density given by (10) we get

$$
\begin{aligned}
\mathrm{E}\left(X^{r}\right) & =\Gamma(1+r / \beta) \sum_{k, l=0}^{\infty} w_{k l} \frac{1}{(\alpha(k+l))^{r / \beta}}, \\
M_{X}(t) & =\sum_{k, l=0}^{\infty} w_{k l} \sum_{r=0}^{\infty} \frac{t^{r}(\alpha(k+l))^{-r / \beta}}{r !} \Gamma(1+r / \beta) \quad \beta \geq 1 .
\end{aligned}
$$

\section{Quantile function and simulation}

The quantile function corresponding to the BTW distribution with cdf (5) is

$$
x=Q(y)=F^{-1}(y)=\left[-\frac{1}{\alpha} \log \left(z^{*}\right)\right]^{1 / \beta},
$$

where $z^{*} \in(0,1)$ is a solution to the quadratic equation

$$
\lambda z^{2}+(1-\lambda) z-\left(1-I_{y}^{-1}(a, b)\right)=0,
$$

and $I_{y}^{-1}(a, b)$ denotes the inverse of the incomplete beta function with parameters $a$ and $b$. Clearly,

$$
z^{*}=\frac{1}{2 \lambda}\left\{\sqrt{(1-\lambda)^{2}+4 \lambda\left(1-I_{y}^{-1}(a, b)\right)}-(1-\lambda)\right\} .
$$

The following expansion for the inverse of the beta incomplete function $I_{y}^{-1}(a, b)$ can be found on the Wolfram website http://functions. wolfram.com/06.23.06.0004.01

$$
\begin{aligned}
I_{u}^{-1}(a, b)= & w+\frac{b-1}{a+1} w^{2}+\frac{(b-1)\left(a^{2}+3 a b-a+5 b-4\right)}{2(a+1)^{2}(a+2)} w^{3} \\
& +\frac{(b-1)\left[a^{4}+(6 b-1) a^{3}+(b+2)(8 b-5) a^{2}\right]}{2(a+1)^{2}(a+2)} w^{4} \\
& +\frac{(b-1)\left[\left(33 b^{2}-30 b+4\right) a+b(31 a-47)+18\right]}{3(a+1)^{3}(a+2)(a+3)} w^{4}+O\left(p^{5 / a}\right),
\end{aligned}
$$

where $w=\{a B(a, b) y\}^{1 / a}, a>0$.

Simulation of $X$ is straightforward from (13) by taking

$$
X=\left[-\frac{1}{\alpha} \log \left(\frac{\sqrt{(1-\lambda)^{2}+4 \lambda(1-B)}-(1-\lambda)}{2 \lambda}\right)\right]^{1 / \beta}
$$

where $B$ is a beta variate with shape parameters $a$ and $b$.

\section{Mean deviation}

The amount of scatter in a population is evidently measured to some extent by the totality of deviations from the mean and the median. If $X$ has a BTW distribution, then we can derive the mean deviations 
about the mean $\mu=\mathrm{E}(X)$ and about the median $M$ as

$$
\begin{aligned}
& \eta_{1}=\int_{0}^{\infty}|x-\mu| f(x) d x, \\
& \eta_{2}=\int_{0}^{\infty}|x-M| f(x) d x .
\end{aligned}
$$

The mean of the distribution is obtained from (11) by putting $r=1$, and the median is obtained by solving the equation

$$
I_{\left(1-\exp \left(-\alpha x^{\beta}\right)\right)\left(1+\lambda \exp \left(-\alpha x^{\beta}\right)\right)}(a, b)=\frac{1}{2} .
$$

Thus, the above measures can be derived from the following relations:

$$
\eta_{1}=2[\mu F(\mu)-J(\mu)] \quad \text { and } \quad \eta_{2}=\mu-2 J(M),
$$

where $J(t)=\int_{0}^{t} x f(x) d x$. From (10) we have

$$
\begin{aligned}
J(t) & =\sum_{k, l=0}^{\infty} w_{k l} \int_{0}^{t} \alpha(k+l) \beta x^{\beta} \exp \left(-\alpha(k+l) x^{\beta}\right) d x \\
& =\sum_{k, l=0}^{\infty} \frac{w_{k l}}{\{\alpha(k+l)\}^{1 / \beta}} \int_{0}^{\alpha(k+l) t^{\beta}} z^{1 / \beta} \exp (-z) d z \\
& =\sum_{k, l=0}^{\infty} \frac{w_{k l}}{\{\alpha(k+l)\}^{1 / \beta}} \gamma\left(\alpha(k+l) t^{\beta}, \beta^{-1}+1\right),
\end{aligned}
$$

where $\gamma(x, \delta)=\int_{0}^{x} w^{\delta-1} \exp (-w) d w, \delta>0$ is an incomplete gamma function. Using (9), one can easily find $\eta_{1}$ and $\eta_{2}$ from (16).

The quantity $J(t)$ can also be used to determine Bonferroni and Lorenz curves, which have applications in economics to study income and poverty, and also in other fields like reliability, demography, insurance and medicine. Bonferroni and Lorenz functions are given by $B(\pi)=J(p) /(\pi \mu)$ and $L(\pi)=J(p) / \mu$, respectively, where $p=Q(\pi)$ is calculated from (13) for a given probability $\pi$.

\section{Order statistics}

If $X_{(1)}<\cdots<X_{(n)}$ denote the ordered observations in a data set from the BTW distribution given by (5) and (6), then the pdf $f_{i: n}(x)$ of the $i$ th order statistic $X_{(i)}$ is

$$
f_{i: n}(x)=\frac{1}{B(i, n-i+1)} f(x) F(x)^{i-1}[1-F(x)]^{n-1} .
$$

Using expressions (9) and (10) for $F(x)$ and $f(x)$, respectively, and applying the binomial expansion yields

$$
\begin{aligned}
f_{i: n}(x)= & \frac{1}{B(i, n-i+1)} f(x) \sum_{s=0}^{n-i}(-1)^{s}\left(\begin{array}{c}
n-i \\
s
\end{array}\right) F(x)^{i+s-1} \\
= & \frac{\alpha \beta x^{\beta-1}}{B(i, n-i+1)}\left(\sum_{k, l=0}^{\infty} w_{k l}(k+l) \exp \left(-\alpha(k+l) x^{\beta}\right)\right) \\
& \sum_{s=0}^{n-i}(-1)^{s+1}\left(\begin{array}{c}
n-i \\
s
\end{array}\right)\left(\sum_{k, l=0}^{\infty} w_{k l} \exp \left(-\alpha(k+l) x^{\beta}\right)\right)^{i+s-1}
\end{aligned}
$$


Writing $u=\exp \left(-\alpha x^{\beta}\right), f_{i: n}(x)$ can be expressed as

$$
\begin{aligned}
f_{i: n}(x)= & \frac{\alpha \beta x^{\beta-1}}{B(i, n-i+1)}\left(\sum_{k, l=0}^{\infty} w_{k l}(k+l) u^{k+l}\right) \\
& \sum_{s=0}^{n-i}(-1)^{s+1}\left(\begin{array}{c}
n-i \\
s
\end{array}\right)\left(\sum_{k, l=0}^{\infty} w_{k l} u^{k+l}\right)^{i+s-1} .
\end{aligned}
$$

We note that in (19) we can write

$$
\sum_{k, l=0}^{\infty} w_{k l} u^{k+l}=\sum_{m=0}^{\infty} w_{m}^{*} u^{m}
$$

and

$$
\sum_{k, l=0}^{\infty} w_{k l}(k+l) u^{k+l}=\sum_{m=0}^{\infty} m w_{m}^{*} u^{m}
$$

where $w_{m}^{*}=\sum_{k, l: k+l=m} w_{k l}$. Further, from (Gradshteyn and Ryzhik 2000, Section 0.314), for any positive integer $r$,

$$
\left(\sum_{k=0}^{\infty} a_{k} u^{k}\right)^{r}=\sum_{k=0}^{\infty} d_{r, k} u^{k}
$$

where the coefficients $d_{r, k}$, for $k=1,2, \ldots$, can be determined from the recurrence equation

$$
d_{r, k}=\left(k a_{0}\right)^{-1} \sum_{m=1}^{k}\{m(r+1)-k\} a_{m} d_{r, k-m}
$$

and $d_{r, 0}=a_{0}^{r}$. Hence, $d_{r, k}$ comes directly from $d_{r, 0}, \ldots, d_{r, k-1}$ and, therefore, from $a_{0}, \ldots, a_{k}$. Using (20) and (21) it follows that

$$
f_{i: n}(x)=\frac{\alpha \beta x^{\beta-1}}{B(i, n-i+1)}\left(\sum_{m=0}^{\infty} m w_{m}^{*} u^{m}\right) \sum_{s=0}^{n-i}(-1)^{s+1}\left(\begin{array}{c}
n-i \\
s
\end{array}\right)\left(\sum_{m=0}^{\infty} d_{i+s-1, m} u^{m}\right),
$$

where

$$
\begin{aligned}
d_{i+s-1, m} & =\left(m w_{0}^{*}\right)^{-1} \sum_{q=1}^{m}[q(i+s)-m] w_{m}^{*} d_{i+s-1, m-q} \\
d_{i+s-1,0} & =\left(w_{0}^{*}\right)^{i+s-1}=\left(\sum_{j=0}^{\infty}(-1)^{j+1}\left(\begin{array}{c}
b-1 \\
j
\end{array}\right) \frac{1}{B(a, b)(a+j)}\right)^{i+s-1} .
\end{aligned}
$$

Combining terms, we obtain

$$
\begin{aligned}
f_{i: n}(x)= & \frac{\alpha \beta x^{\beta-1}}{B(i, n-i+1)} \sum_{s=0}^{n-i}(-1)^{s+1}\left(\begin{array}{c}
n-i \\
s
\end{array}\right) \sum_{m=1}^{\infty} \sum_{t=0}^{\infty} m d_{i+s-1, t} w_{m}^{*} u^{m+t} \\
= & \frac{1}{B(i, n-i+1)} \sum_{s=0}^{n-i}(-1)^{s+1}\left(\begin{array}{c}
n-i \\
s
\end{array}\right) \\
& \sum_{m=1}^{\infty} \sum_{t=0}^{\infty} \frac{m d_{i+s-1, t} w_{m}^{*}}{m+t}\left\{(m+t) \alpha \beta x^{\beta-1} \exp \left(-(m+t) \alpha x^{\beta}\right)\right\} \\
= & \sum_{m=1}^{\infty} \sum_{t=0}^{\infty} c_{i}(m, t) g(x ;(m+t) \alpha, \beta)
\end{aligned}
$$


where $g(x ;(m+t) \alpha, \beta)$ denotes the pdf of a Weibull distribution with scale parameter $(m+t) \alpha$ and shape parameter $\beta$ and

$$
c_{i}(m, t)=\frac{1}{B(i, n-i+1)} \frac{m w_{m}^{*}}{m+t} \sum_{s=0}^{n-i}(-1)^{s+1}\left(\begin{array}{c}
n-i \\
s
\end{array}\right) d_{i+s-1, t} .
$$

\section{Moments of order statistics and L-moments}

The moments of the order statistics of BTW distribution can be easily written in terms of those of a Weibull distribution by using the expression (22) of the pdf of the order statistic distribution. We get

$$
\mathrm{E}\left(X_{i: n}^{r}\right)=\Gamma\left(\frac{r}{\beta+1}\right) \sum_{m=1}^{\infty} \sum_{t=0}^{\infty} c_{i}(m, t)\{(m+t) \alpha\}^{-r / \beta},
$$

where $c_{i}(m, t)$ is given in (23).

As indicated by Cordeira et al. (2013), L-moments Hosking (1990) are summary statistics for probability distributions and data samples but have several advantages over ordinary moments. For example, they apply for any distribution having a finite mean and no higher-order moments need be finite. The $r$ th L-moment is computed from linear combinations of the ordered data values by

$$
\rho_{r}=\sum_{j=0}^{r-1}(-1)^{r-j-1}\left(\begin{array}{c}
r-1 \\
j
\end{array}\right)\left(\begin{array}{c}
r+j-1 \\
j
\end{array}\right) \gamma_{j}
$$

where $\gamma_{j}=\mathrm{E}\left(X F(X)^{j}\right)$. Thus, $\rho_{1}=\gamma_{0}, \rho_{2}=2 \gamma_{1}-\gamma_{0}, \rho_{3}=6 \gamma_{2}-6 \gamma_{1}+\gamma_{0}$, and $\rho_{4}=$ $20 \gamma_{3}-30 \gamma_{2}+12 \gamma_{1}-\gamma_{0}$. In general, we get $\gamma_{k}=(k+1)^{-1} \mathrm{E}\left(X_{k+1: k+1}\right)$, which can be computed from (24) by using (23) and putting $i=n=k+1$ and $r=1$.

\section{Reliability}

A stress-strength model describes the life of a component which has a random strength $X_{1}$ and is subjected to a random stress $X_{2}$. The component functions satisfactorily as long as $X_{1}>X_{2}$, and fails when $X_{1}<X_{2}$. The probability $R=\operatorname{Pr}\left(X_{1}>X_{2}\right)$ defines the component reliability. Stressstrength models have many applications especially in engineering concepts such as structures, deterioration of rocket motors, static fatigue of ceramic components, fatigue failure of aircraft structures and the aging of concrete pressure vessels.

Consider $X_{1}$ and $X_{2}$ to be independently distributed, with $X_{1} \sim \operatorname{BTW}\left(\alpha_{1}, \beta, \lambda_{1}, a_{1}, b_{1}\right)$ and $X_{2} \sim$ $\operatorname{BTW}\left(\alpha_{2}, \beta, \lambda_{2}, a_{2}, b_{2}\right)$.The cdf $F_{1}$ of $X_{1}$ and pdf $f_{2}$ of $X_{2}$ are obtained from (9) and (10), respectively. Then,

$$
\begin{aligned}
R=\operatorname{Pr}\left(X_{1}>X_{2}\right) & =\int_{0}^{\infty} f_{2}(y)\left[1-F_{1}(y)\right] d y \\
& =1+\sum_{k, l=0}^{\infty} w_{k l}^{(1)} \int_{0}^{\infty} f_{2}(y) \exp \left(-\alpha(k+l) y^{\beta}\right) d y \\
& =\sum_{k, l=0}^{\infty} w_{k l}^{(1)} A(k, l),
\end{aligned}
$$

where

$$
w_{k l}^{(i)}=\sum_{j=0}^{\infty}(-1)^{j+k+1}\left(\begin{array}{c}
b_{i}-1 \\
j
\end{array}\right)\left(\begin{array}{c}
a_{i}+j \\
k
\end{array}\right)\left(\begin{array}{c}
a_{i}+j \\
l
\end{array}\right) \frac{\lambda^{l}}{B\left(a_{i}, b_{i}\right)\left(a_{i}+j\right)}, \quad i=1,2,
$$


and

$$
A(k, l)=\int_{0}^{\infty} f_{2}(y) \exp \left(-\alpha(k+l) y^{\beta}\right) d y .
$$

Now,

$$
\begin{aligned}
A(k, l) & =\sum_{r, s=0}^{\infty} w_{r s}^{(2)} \int_{0}^{\infty}(r+s) \alpha_{2} \beta y^{\beta-1} \exp \left[-\left\{\alpha_{1}(k+l)+(r+s) \alpha_{2}\right\} y^{\beta}\right] d y \\
& =\sum_{r, s=0}^{\infty} w_{r s}^{(2)} \frac{(r+s) \alpha_{2}}{\alpha_{1}(k+l)+(r+s) \alpha_{2}}
\end{aligned}
$$

Hence,

$$
\begin{aligned}
R & =1+\sum_{k, l=0}^{\infty} w_{k l}^{(1)} \sum_{r, s=0}^{\infty} w_{r s}^{(2)} \frac{(r+s) \alpha_{2}}{(k+l) \alpha_{1}+(r+s) \alpha_{2}} \\
& =1+\sum_{k=0}^{\infty} \sum_{r=0}^{\infty} w_{k}^{*(1)} w_{r}^{*(2)} \frac{r \alpha_{2}}{k \alpha_{1}+r \alpha_{2}},
\end{aligned}
$$

where

$$
w_{m}^{*(i)}=\sum_{k, l: k+l=m} w_{k l}^{(i)}, \quad i=1,2 .
$$

\section{Maximum likelihood estimation}

Let $\theta=(\alpha, \beta, \lambda, a, b)$ denote the parameter vector for the BTW distribution with pdf given by (6). Then, the log-likelihood function $\ell(\theta)$ based on a single observation $x$ is

$$
\begin{aligned}
\ell(\theta)= & \log (\alpha)+\log (\beta)-\log B(a, b)+(\beta-1) \log (x)-\alpha x^{\beta}+\log \left(1-\lambda+2 \lambda \exp \left(-\alpha x^{\beta}\right)\right) \\
& +(a-1)\left\{\log \left(1-\exp \left(-\alpha x^{\beta}\right)\right)+\log \left(1+\lambda \exp \left(-\alpha x^{\beta}\right)\right)\right\} \\
& +(b-1) \log \left\{1-\left(1-\exp \left(-\alpha x^{\beta}\right)\right)\left(1+\lambda \exp \left(-\alpha x^{\beta}\right)\right)\right\} .
\end{aligned}
$$

Hence, the components of the unit score vector

$$
\frac{\partial \ell(\theta)}{\partial \theta}=\left(\frac{\partial \ell(\theta)}{\partial \alpha}, \frac{\partial \ell(\theta)}{\partial \beta}, \frac{\partial \ell(\theta)}{\partial \lambda}, \frac{\partial \ell(\theta)}{\partial a}, \frac{\partial \ell(\theta)}{\partial b}\right)^{\prime}
$$

are

$$
\begin{gathered}
\frac{\partial \ell(\theta)}{\partial \alpha}=\frac{1}{\alpha}-x^{\beta}-\frac{2 \lambda x^{\beta} \exp \left(-\alpha x^{\beta}\right)}{1-\lambda+2 \lambda \exp \left(-\alpha x^{\beta}\right)}+\frac{(a-1) x^{\beta} \exp \left(-\alpha x^{\beta}\right)}{1-\exp \left(-\alpha x^{\beta}\right)}-\frac{(a-1) \lambda x^{\beta} \exp \left(-\alpha x^{\beta}\right)}{1+\lambda \exp \left(-\alpha x^{\beta}\right)} \\
-\frac{(b-1) x^{\beta} \exp \left(-\alpha x^{\beta}\right)}{1-\left(1-\exp \left(-\alpha x^{\beta}\right)\right)\left(1+\lambda \exp \left(-\alpha x^{\beta}\right)\right)}\left(1-\lambda+2 \lambda \exp \left(-\alpha x^{\beta}\right)\right) \\
\frac{\partial \ell(\theta)}{\partial \beta}=\frac{1}{\beta}+\log (x)-\alpha x^{\beta} \log (x)-\frac{2 \lambda \alpha x^{\beta} \log (x) \exp \left(-\alpha x^{\beta}\right)}{1-\lambda+2 \lambda \exp \left(-\alpha x^{\beta}\right)} \\
\quad+\frac{(a-1) x^{\beta} \log (x) \exp \left(-\alpha x^{\beta}\right)}{1-\exp \left(-\alpha x^{\beta}\right)}-\frac{(a-1) \lambda x^{\beta} \log (x) \exp \left(-\alpha x^{\beta}\right)}{1+\lambda \exp \left(-\alpha x^{\beta}\right)} \\
\quad-\frac{(b-1) x^{\beta} \log (x) \exp \left(-\alpha x^{\beta}\right)}{1-\left(1-\exp \left(-\alpha x^{\beta}\right)\right)\left(1+\lambda \exp \left(-\alpha x^{\beta}\right)\right)}\left(1-\lambda+2 \lambda \exp \left(-\alpha x^{\beta}\right)\right)
\end{gathered}
$$




$$
\begin{aligned}
& \frac{\partial \ell(\theta)}{\partial \lambda}=-\frac{1-2 \exp \left(-\alpha x^{\beta}\right)}{1-\lambda+2 \lambda \exp \left(-\alpha x^{\beta}\right)}+\frac{(a-1) \exp \left(-\alpha x^{\beta}\right)}{1+\lambda \exp \left(-\alpha x^{\beta}\right)} \\
&-\frac{(b-1) \exp \left(-\alpha x^{\beta}\right)\left(1-\exp \left(-\alpha x^{\beta}\right)\right)}{1-\left(1-\exp \left(-\alpha x^{\beta}\right)\right)\left(1+\lambda \exp \left(-\alpha x^{\beta}\right)\right)} \\
& \frac{\partial \ell(\theta)}{\partial a}=\Psi(a+b)-\Psi(a)+\left\{\log \left(1-\exp \left(-\alpha x^{\beta}\right)\right)+\log \left(1+\lambda \exp \left(-\alpha x^{\beta}\right)\right)\right\} \\
& \frac{\partial \ell(\theta)}{\partial b}=\Psi(a+b)-\Psi(b)+\log \left\{1-\left(1-\exp \left(-\alpha x^{\beta}\right)\right)\left(1+\lambda \exp \left(-\alpha x^{\beta}\right)\right)\right\},
\end{aligned}
$$

where $\Psi(x)=\frac{d}{d x} \log \Gamma(x)$.

For a random sample $\left(x_{1}, \ldots, x_{n}\right)$ of size $n$ from $X$, distributed with pdf (6), the sample loglikelihood is $\ell(\theta)=\sum_{i=0}^{n} \ell_{i}(\theta)$, where $\ell_{i}(\theta)$ is the log-likelihood for the $i$ th observation $(i=$ $1, \ldots, n)$, and the score vector is

$$
\frac{\partial \ell(\theta)}{\partial \theta}=\sum_{i=0}^{n} \frac{\partial \ell_{i}(\theta)}{\partial \theta}
$$

The maximum likelihood estimate (MLE) $\hat{\theta}$ of $\theta$ is obtained by solving the system

$$
\frac{\partial \ell(\theta)}{\partial \theta}=0
$$

Under certain regularity conditions, $\sqrt{n}(\hat{\theta}-\theta) \stackrel{d}{\rightarrow} \operatorname{Normal}\left(0, I^{-1}(\theta)\right)$ (here $\stackrel{d}{\rightarrow}$ stands for convergence in distribution), where $I(\theta)$ denotes the information matrix given by

$$
I(\theta)=\mathrm{E}\left(\frac{\partial^{2} \ell(\theta)}{\partial \theta \partial \theta^{\prime}}\right)
$$

This information matrix $I(\theta)$ may be approximated by the observed information matrix

$$
I(\hat{\theta})=\left.\left(\frac{\partial^{2} \ell(\theta)}{\partial \theta \partial \theta^{\prime}}\right)\right|_{\theta=\hat{\theta}} .
$$

Then, using the approximation $\sqrt{n}(\hat{\theta}-\theta) \sim \operatorname{Normal}\left(0, I^{-1}(\hat{\theta})\right)$, one can carry out tests and find confidence regions for functions of some or all parameters in $\theta$.

\section{The log beta transmuted Weibul distribution}

If $X$ is a random variable having the BTW distribution given by (8), then $Y=\log (X)$ is said to have a log beta transmuted Weibull (LBTW) distribution.

Let us define $\mu=-1 / \beta \log (\alpha)$. Then, the pdf of $Y$ is given by

$$
\begin{aligned}
f_{Y}(y)= & \frac{\beta}{B(a, b)} \exp \{\beta(y-\mu)-\exp (\beta(y-\mu))\}\{1-\lambda+2 \lambda \exp (-\exp (\beta(y-\mu)))\} \\
& \{1-\exp (-\exp (\beta(y-\mu)))\}^{a-1}\{1+\lambda \exp (-\exp (\beta(y-\mu)))\}^{a-1} \\
& {[1-\{1-\exp (-\exp (\beta(y-\mu)))\}\{1+\lambda \exp (-\exp (\beta(y-\mu)))\}]^{b-1}, }
\end{aligned}
$$

where $y, \mu \in \mathbb{R}$ and $\beta>0$. Its corresponding cdf is

$$
F_{Y}(y)=I_{A(y)}(a, b), \quad y \in \mathbb{R},
$$

where

$$
A(y)=\{1-\exp (-\exp (\beta(y-\mu)))\}\{1+\lambda \exp (-\exp (\beta(y-\mu)))\}
$$


The standardized random variable $Z=\beta(Y-\mu)$, therefore, has pdf

$$
\begin{aligned}
f_{Z}(z)= & \frac{1}{B(a, b)} \exp \{z-\exp (z)\}\{1-\lambda+2 \lambda \exp (-\exp (z))\} \\
& \{1-\exp (-\exp (z))\}^{a-1}\{1+\lambda \exp (-\exp (z))\}^{a-1} \\
& {[1-\{1-\exp (-\exp (z))\}\{1+\lambda \exp (-\exp (z))\}]^{b-1}, \quad z \in \mathbb{R} . }
\end{aligned}
$$

For $a=b=1$, we get the log-transmuted Weibull distribution, while for $a=b=1$ and $\lambda=0$ we get the log-Weibull distribution or the extreme value distribution.



Figure 3: Plots of LBTW pdf's for increasing $\lambda$ and $a$, when $b=0.5, \mu=0$, and $\alpha=1$.

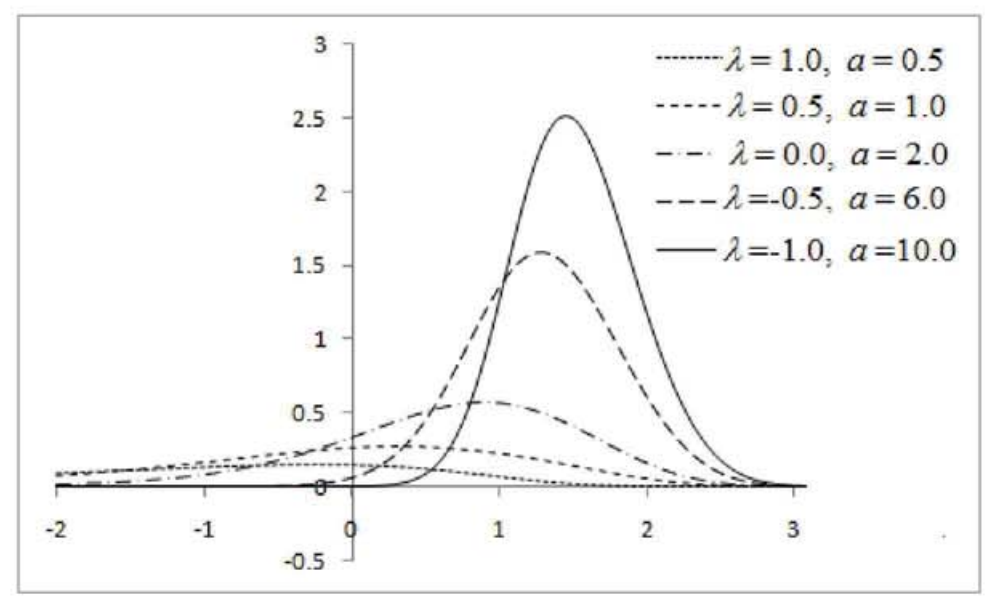

Figure 4: Plots of LBTW pdf's for $\lambda$ decreasing and $a$ increasing, when $b=0.5, \mu=0$, and $\alpha=1$.

The $r$ th moment of the standardized distribution (28) is given by

$$
\begin{aligned}
\mathrm{E}\left(Z^{r}\right)= & \int_{-\infty}^{\infty} z^{r} f_{Z}(z) d z \\
= & \frac{1}{B(a, b)} \sum_{i=0}^{\infty}(-1)^{i}\left(\begin{array}{c}
b-1 \\
i
\end{array}\right) \int_{-\infty}^{\infty} z^{r} \exp [z-\exp (z)][1-\lambda+2 \lambda \exp \{-\exp (z)\}] \\
& {[1-\exp \{-\exp (z)\}]^{a+i-1}[1+\lambda \exp \{-\exp (z)\}]^{a+i-1} d z, }
\end{aligned}
$$

using the binomial expansion. 


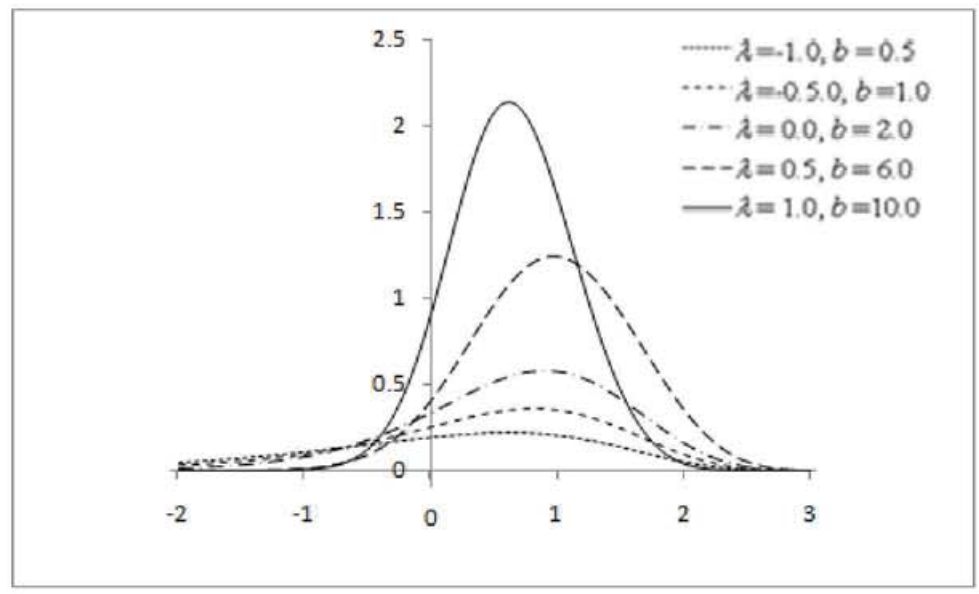

Figure 5: Plots of LBTW densities for increasing $\lambda$ and increasing $b$, when $a=0.5, \mu=0$, and $\alpha=1$.

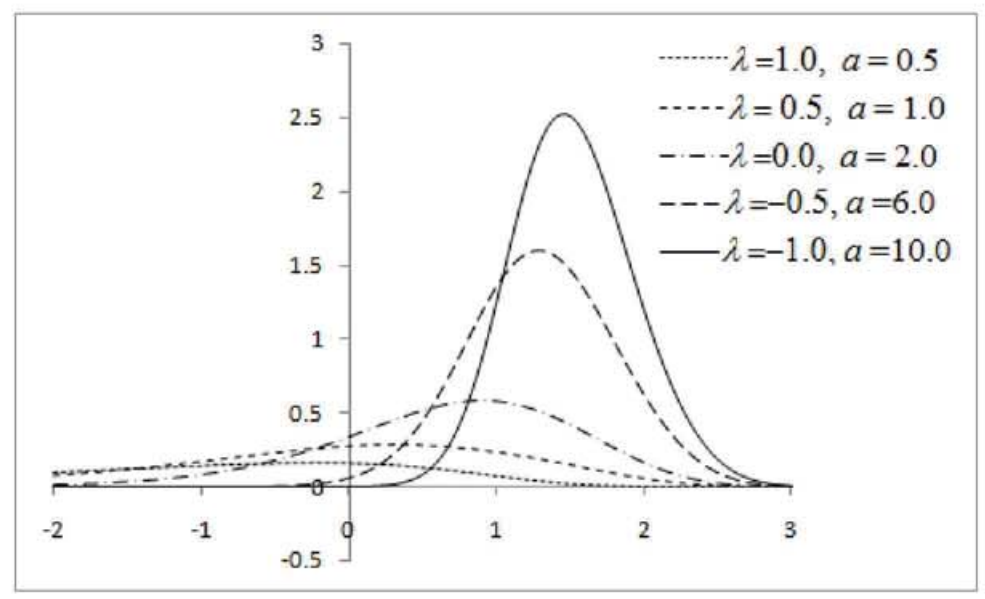

Figure 6: Plots of LBTW pdf's for decreasing $\lambda$ and increasing $b$, when $a=0.5, \mu=0$, and $\alpha=1$.

Setting $u=\exp (z)$ we get

$$
\begin{aligned}
\mathrm{E}\left(Z^{r}\right)= & \frac{1}{B(a, b)} \sum_{i=0}^{\infty}(-1)^{i}\left(\begin{array}{c}
b-1 \\
i
\end{array}\right) \\
& \int_{-\infty}^{\infty} \log (u)^{r} \exp (-u)[1-\lambda+2 \lambda \exp \{-u\}] \\
& {[1-\exp \{-u\}]^{a+i-1}[1+\lambda \exp \{-u\}]^{a+i-1} d u . }
\end{aligned}
$$

By further power series expansion of binomial, we then have

$$
\begin{aligned}
\mathrm{E}\left(Z^{r}\right)= & \frac{1}{B(a, b)} \sum_{i, j, k=0}^{\infty}(-1)^{i+j} \lambda^{k}\left(\begin{array}{c}
b-1 \\
i
\end{array}\right)\left(\begin{array}{c}
a+i-1 \\
j
\end{array}\right)\left(\begin{array}{c}
a+i-1 \\
k
\end{array}\right) \\
& \int_{-\infty}^{\infty} \log (u)^{r} \exp [-(j+k+1) u][1-\lambda+2 \lambda \exp \{-u\}] d u .
\end{aligned}
$$

By (Prudnikov, Brychkov, and Marichev 1986, equation 2.6.21.1), we have

$$
I(r, s)=\int_{-\infty}^{\infty} \log (u)^{r} \exp (-s u) d u=\left.\left(\frac{\partial^{r} s^{-p} \Gamma(p)}{\partial p^{r}}\right)\right|_{p=1} .
$$


Using (30) in (29), we thus obtain

$$
\begin{aligned}
\mathrm{E}\left(Z^{r}\right)= & \frac{1}{B(a, b)} \sum_{i, j, k=0}^{\infty}(-1)^{i+j} \lambda^{k}\left(\begin{array}{c}
b-1 \\
i
\end{array}\right)\left(\begin{array}{c}
a+i-1 \\
j
\end{array}\right)\left(\begin{array}{c}
a+i-1 \\
k
\end{array}\right) \\
& {[(1-\lambda) I(r, j+k+1)+2 \lambda I(r, j+k+2)] . }
\end{aligned}
$$

In many practical situations, the value of a random variable is affected by the values of a number of other variables, called explanatory variables. For example, if $X$ denotes the lifetime of a system, then it is affected by explanatory variables like lifetimes of its sub-components, surrounding temperature, etc.

Consider a type 1 censored sample of size $n$, where $x_{i}$ denotes the true lifetime and $c_{i}$ the censoring time of the $i$ th sampled unit, and $v_{i}=\left(v_{1 i}, \ldots, v_{p i}\right)^{\prime}$ denotes the corresponding vector of explanatory variables, $i=1, \ldots, n$. The $i$ th response $y_{i}$ is defined as $y_{i}=\min \left[\log \left(x_{i}\right), \log \left(c_{i}\right)\right]$. Consider a linear regression model for the response variable using LBTW distribution as follows:

$$
y_{i}=v_{i}^{\prime} \gamma+\frac{1}{\beta} z_{i}, \quad i=1, \ldots, n,
$$

where the $z_{i}$ 's are independently distributed with density (28), $\gamma=\left(\gamma_{1}, \ldots, \gamma_{p}\right)^{\prime},|\lambda| \leq 1, a>0$, $b>0$, and the location parameter $\mu_{i}$ corresponding to the $i$ th lifetime is modeled as $\mu_{i}=v_{i}^{\prime} \gamma$. Thus, the location vector for the LBTW model has the structure $\mu=v^{\prime} \gamma$, where $\mu=\left(\mu_{1}, \ldots, \mu_{n}\right)^{\prime}$ and $v=\left(v_{1}, \ldots, v_{n}\right)^{\prime}$. For $a=b=1$, the model reduces to the log-transmuted Weibull model, while for $\lambda=0$ and $a=b=1$ it reduces to the log-Weibull (or the extreme value) model.

Denoting by $C$ and $N$ the sets of indices for the censored and uncensored observations respectively, the $\log$-likelihood for the model parameters $\theta=\left(\beta, \lambda, a, b, \gamma^{\prime}\right)^{\prime}$ is given by

$$
\begin{aligned}
\ell(\theta)= & q[\log (\beta)-\log \{B(a, b)\}] \\
& +\sum_{i \in N}\left[\beta\left(y_{i}-\mu_{i}\right)-\exp \left(\beta\left(y_{i}-\mu_{i}\right)\right)+\log \left\{1+\lambda \exp \left(-\exp \left(\beta\left(y_{i}-\mu_{i}\right)\right)\right)\right\}\right] \\
& +(a-1) \sum_{i \in N}\left[\log \left\{1-\exp \left(-\exp \left(\beta\left(y_{i}-\mu_{i}\right)\right)\right)\right\}+\log \left\{1+\lambda \exp \left(-\exp \left(\beta\left(y_{i}-\mu_{i}\right)\right)\right)\right\}\right] \\
& +(b-1) \sum_{i \in N}\left[\log \left\{1-\left\{1-\exp \left(-\exp \left(\beta\left(y_{i}-\mu_{i}\right)\right)\right)\right\}\left\{1+\lambda \exp \left(-\exp \left(\beta\left(y_{i}-\mu_{i}\right)\right)\right)\right\}\right\}\right] \\
& +\sum_{i \in C} \log \left[1-I_{\left\{1-\exp \left(-\exp \left(\beta\left(c_{i}-\mu_{i}\right)\right)\right)\right\}\left\{1+\lambda \exp \left(-\exp \left(\beta\left(c_{i}-\mu_{i}\right)\right)\right)\right\}}(a, b)\right]
\end{aligned}
$$

where $q$ is the number of observed failures. The MLE $\hat{\theta}$ of $\theta$ is obtained by solving the likelihood equations $\partial \ell(\theta) / \partial \theta=0$.

Under certain regularity conditions, the centered form of the MLE, $\sqrt{n}(\hat{\theta}-\theta)$, is asymptotically distributed as $\operatorname{Normal}\left(0, K^{-1}(\theta)\right)$, where $K(\theta)$ is the information matrix, given by

$$
K(\theta)=\mathrm{E}\left(\frac{\partial^{2} \ell(\theta)}{\partial \theta \partial \theta^{\prime}}\right)
$$

and can be approximated by $K(\hat{\theta})$. Then, based on $\sqrt{n}(\hat{\theta}-\theta) \sim \operatorname{Normal}\left(0, K^{-1}(\hat{\theta})\right)$, one can carry out tests and find confidence regions for functions of $\theta$.

\section{Simulation study}

A simulation study is carried out to investigate the performance of the MLEs. We take sample sizes to be $n \in\{15,25,50\}$, and generate observations from a BTW distribution with parameters $\alpha=1$, $\beta=2, \lambda=0.5, a=b=2$.The MLEs and 95\% confidence intervals are computed using the observed Fisher information matrix. The process is replicated 1000 times, and the average estimates, along with 
Table 1: Average MLEs of the parameters and the corresponding mean squared errors (in parenthesis).

\begin{tabular}{cccccc}
\hline \multicolumn{5}{c}{ MLEs } \\
$n$ & $\hat{\alpha}$ & $\hat{\beta}$ & $\hat{\lambda}$ & $\hat{a}$ & $\hat{b}$ \\
\hline 15 & $0.565(0.201)$ & $1.251(0.229)$ & $0.392(0.242)$ & $1.121(0.198)$ & $1.301(0.213)$ \\
25 & $0.799(0.115)$ & $1.723(0.099)$ & $0.435(0.123)$ & $1.728(0.089)$ & $1.591(0.101)$ \\
50 & $1.027(0.036)$ & $1.923(0.034)$ & $0.485(0.041)$ & $2.013(0.021)$ & $1.935(0.024)$ \\
\hline
\end{tabular}

the mean squared error are presented in Table 1. In Table 2, the average 95\% confidence intervals are reported.

From Table 1 it is observed that as the sample size increases, the average biases and the mean squared errors decrease. This verifies the consistency properties of the estimates.

Table 2: Average $95 \%$ confidence intervals for the parameters.

\begin{tabular}{cccccc}
\hline$n$ & $\alpha$ & $\beta$ & $\lambda$ & $a$ & $b$ \\
\hline 15 & $(0.375,2.075)$ & $(0.617,3.675)$ & $(0.072,1.727)$ & $(0.523,4.026)$ & $(0.576,3.972)$ \\
25 & $(0.477,1.736)$ & $(0.760,3.521)$ & $(0.162,1.216)$ & $(0.727,3.529)$ & $(0.833,3.488)$ \\
50 & $(0.625,1.421)$ & $(1.102,2.648)$ & $(0.199,0.874)$ & $(1.001,3.135)$ & $(0.928,2.846)$ \\
\hline
\end{tabular}

Table 2 shows that as the sample size increases, the average confidence lengths decrease and the intervals tend towards symmetry.

\section{Application of the beta transmuted Weibull model}

In this section we illustrate the usefulness of the beta transmuted Weibull distribution for modeling reliability data, and also give an application of the log beta transmuted Weibull regression model. We consider two real data sets, and, for the former, we compare our results with those obtained by fitting the transmuted Weibull distribution, beta exponentiated Weibull distribution, exponentiated Weibull distribution and the Weibull distribution. The cdf of the beta exponentiated Weibull distribution is given by

$$
F_{B E W}(x)=\frac{1}{B(a, b)} \int_{0}^{\left[1-\exp \left(-\alpha x^{\beta}\right)\right]^{\gamma}} w^{a-1}(1-w)^{b-1} d w, \quad x, \alpha, \beta, \gamma, a, b>0,
$$

where $\gamma$ is the exponentiating parameter. For $a=b=1$, it becomes the exponentiated Weibull distribution, which is a special case of BTW distribution with $\lambda=0, a=\gamma$, and $b=1$. For $a=b=\gamma=1$, (34) reduces to the Weibull distribution.

\subsection{Tensile fatigue characteristics of yarn}

The first data set relates to the time-to-failure of a polyster/viscose yarn in a textile experiment for testing the tensile fatigue characteristics of yarn. It consists of a sample of 100 centimeter yarn at 2.3\% strain level. This data was also studied by Quesenberry and Kent (1982), and is given in Table 3. The Weibull, exponentiated Weibull, beta exponentiated Weibull, transmuted Weibull and beta transmuted Weibull distributions are fitted to the data and the MLEs of the parameters are computed are given in Table 3. The values of maximized log-likelihoods, Akaike information criterion (AIC), Bayesian information criterion (BIC) and Kolmogorov-Smirnov statistic (K-S) for the different fitted distributions are also given. Though all the distributions considered give good fits, the beta transmuted Weibull distribution is seen to be marginally better than the others. A graphical comparison of the fitted models is displayed in Figure 7.

\subsection{Class-H insulation data}

As an application of the LBEW regression model, we consider the accelerated test data given in 
Table 3: Failure time data on $100 \mathrm{cms}$. Yarn at $2.3 \%$ strain level (sample size $n=100$ ).

\begin{tabular}{rrrrrrrrrrrrr}
\hline 86 & 146 & 251 & 653 & 98 & 249 & 400 & 292 & 131 & 169 & 175 & 176 & 76 \\
264 & 15 & 364 & 195 & 262 & 88 & 264 & 157 & 220 & 42 & 321 & 180 & 198 \\
38 & 20 & 61 & 121 & 282 & 224 & 149 & 180 & 325 & 250 & 196 & 90 & 229 \\
166 & 38 & 337 & 65 & 151 & 341 & 40 & 40 & 135 & 597 & 246 & 211 & 180 \\
93 & 315 & 353 & 571 & 124 & 279 & 81 & 186 & 497 & 182 & 423 & 185 & 229 \\
400 & 338 & 290 & 398 & 71 & 246 & 185 & 188 & 568 & 55 & 55 & 61 & 244 \\
20 & 284 & 393 & 396 & 203 & 829 & 239 & 236 & 286 & 194 & 277 & 143 & 198 \\
264 & 105 & 203 & 124 & 137 & 135 & 350 & 193 & 188 & & & & \\
\hline
\end{tabular}

Table 4: Estimated parameters of the Weibull (W), exponentiated Weibull (EW), beta exponentiated Weibull (BEW), transmuted Weibull (TW) and beta transmuted Weibull (BTW) distributions, and the corresponding values of log-likelihood (LL), Akaike information criterion (AIC), Bayesian information criterion (BIC) and Kolmogorov-Smirnov statistic (K-S).

\begin{tabular}{rcccccccccc}
\hline Distribution & $\hat{\alpha}$ & $\hat{\beta}$ & $\hat{\gamma}$ & $\hat{\lambda}$ & $\hat{a}$ & $\hat{b}$ & LL & AIC & BIC & K-S \\
\hline $\mathrm{W}$ & $1.48 \cdot 10^{-4}$ & 1.60 & 1.00 & 0.00 & 1.00 & 1.00 & -627.05 & 1258.10 & 1263.31 & 0.0700 \\
$\mathrm{EW}$ & $2.63 \cdot 10^{-4}$ & 1.50 & 1.00 & 0.00 & 1.00 & 1.00 & -625.58 & 1257.16 & 1264.98 & 0.0685 \\
$\mathrm{TW}$ & $4.68 \cdot 10^{-5}$ & 1.72 & 1.00 & 0.75 & 1.00 & 1.00 & -624.52 & 1255.04 & 1264.86 & 0.0669 \\
$\mathrm{BEW}$ & $1.75 \cdot 10^{-3}$ & 1.04 & 1.29 & 0.00 & 2.01 & 0.26 & -622.63 & 1255.26 & 1268.28 & 0.0681 \\
$\mathrm{BTW}$ & $6.14 \cdot 10^{-4}$ & 1.45 & 1.00 & 0.99 & 1.29 & 0.27 & -619.80 & 1249.58 & 1262.63 & 0.0659 \\
\hline
\end{tabular}

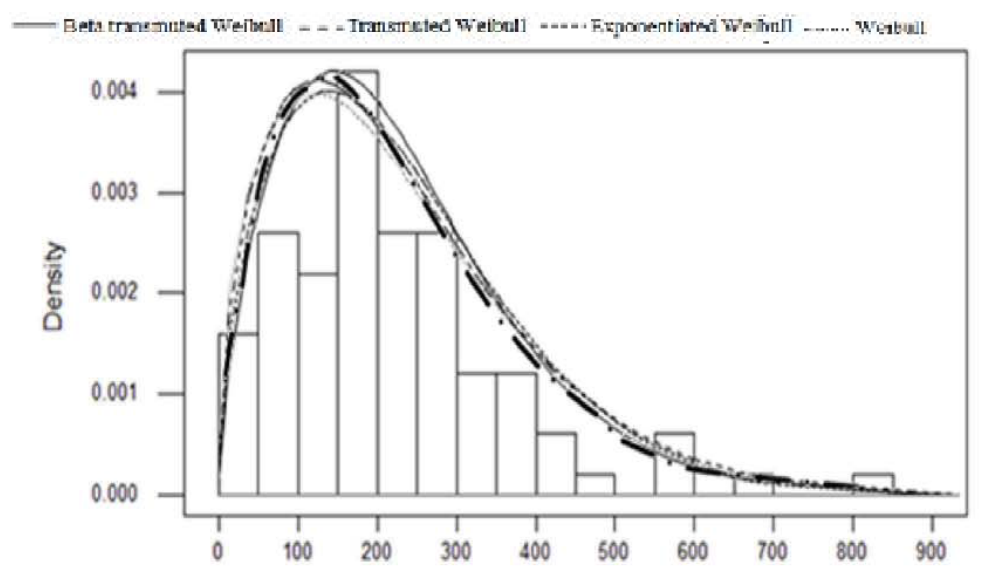

Figure 7: Beta transmuted Weibull, beta exponentiated Weibull, transmuted Weibull, exponentiated Weibull and Weibull densities fitted to the data given in Table 3.

Nelson (1982), which relates to the log time-to-failure of a class $\mathrm{H}$ electrical insulation for motors. Four test temperatures were considered: $190,220,240$ and $260^{\circ} \mathrm{C}$, and a sample of 10 specimens were taken for each test temperature. The specimens were periodically inspected for failure, and the failure time (in hours) of observation $i$, viz. $t_{i}$, was defined as the midpoint of the interval where the failure occurred. Let, $x_{i 1}$ denote the temperature at the $i$ th failure. The data are given in Table 5 below:

We adopt the model

$$
y_{i}=\beta_{0}+\beta_{1} x_{i 1}+\frac{1}{\beta} z_{i},
$$

where the variable $y_{i}=\log t_{i}$ follows the LBTW distribution (28) for $i=1, \ldots, 40$. The MLEs of the model parameters are obtained as $\hat{\beta}=1.2235, \hat{\lambda}=0.2345, \hat{a}=60.25, \hat{b}=1.0210, \hat{\beta}_{0}=11.21$, $\hat{\beta}_{1}=-0.0287$, and the corresponding log-likelihood value is -3.25 . Further, it is noted that from the fitted model that that there is a significant difference between the temperatures levels $190^{\circ} \mathrm{C}$, $220^{\circ} \mathrm{C}, 240^{\circ} \mathrm{C}$, and $260^{\circ} \mathrm{C}$ for the failure times. The curves displayed in Figure 8 represent the empirical survival function and the estimated survival function obtained from (27). It shows that the 
Table 5: Log life of class $\mathrm{H}$ specimens.

\begin{tabular}{cccc}
\hline $190^{\circ} \mathrm{C}$ & $220^{\circ} \mathrm{C}$ & $240^{\circ} \mathrm{C}$ & $260^{\circ} \mathrm{C}$ \\
\hline 3.8590 & 3.2465 & 3.0700 & 2.7782 \\
3.8590 & 3.3867 & 3.0700 & 2.8716 \\
3.8590 & 3.3867 & 3.1821 & 2.8716 \\
3.9268 & 3.3867 & 3.1956 & 2.8716 \\
3.9622 & 3.3867 & 3.2087 & 2.9600 \\
3.9622 & 3.2867 & 3.2214 & 3.0892 \\
3.9622 & 3.4925 & 3.2214 & 3.1206 \\
3.9622 & 3.4925 & 3.2338 & 3.1655 \\
4.0216 & 3.4925 & 3.2458 & 3.2063 \\
4.0216 & 3.4925 & 3.2907 & 3.2778 \\
\hline
\end{tabular}

fit is considerably good.

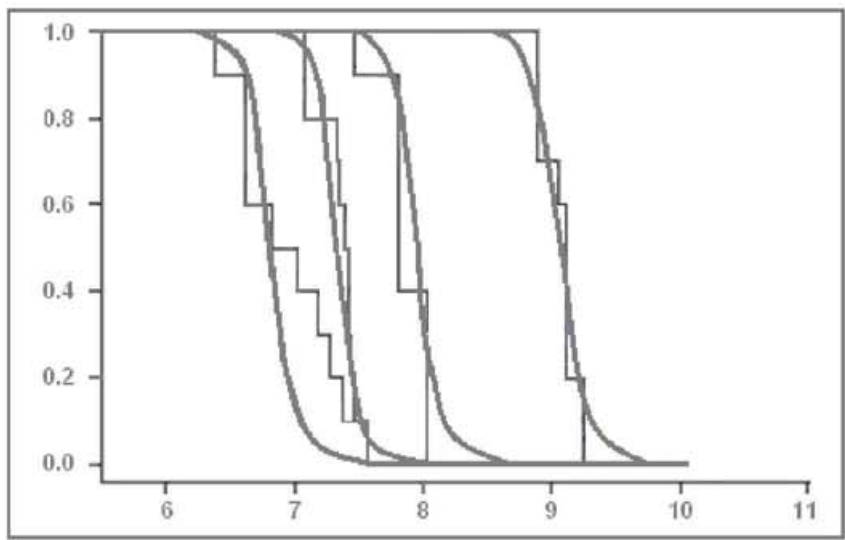

Figure 8: Estimated survival function and the empirical survival function.

\section{Conclusions}

The paper studies some general properties of a new distribution called beta transmuted Weibull distribution. The distribution is a generalization of the Weibull distribution, and includes the Weibull, exponentiated Weibull, exponentiated transmuted Weibull, transmuted Weibull, exponentiated exponential and the exponential distributions as special cases. The log beta transmuted Weibull model has also been discussed, which is appropriate for modeling censored data. Applications of the models to real-life data have been cited and shown to give considerable good fits.

\section{Acknowledgements}

The authors thank the anonymous referee for the valuable comments, which helped immensely to improve the presentation.

\section{References}

Aryall GR, Tsokos CP (2011). "Transmuted Weibull Distribution: A Generalization of the Weibull Probability Distribution." European Journal of Pure and Applied Mathematics, 4, 89-102.

Cordeira GM, Gomes AE, da-Silva CQ, Ortega EMM (2013). "The Beta Exponentiated Weibull Distribution.” Journal of Statistical Computation and Simulation, 83, 114-138. 
Cordeiro GM, Nadarajah S (2011). "Closed form Expressions for Moments of a Class of Beta Generalized Distributions.” Brazilian Journal of Probability and Statistics, 25, 14-33.

Elbatal I (2011). "Exponentiated Modified Weibull Distribution.” Economic Quality Control, 26, 189-200.

Eugene N, Lee C, Famoye F (2002). "Beta-normal Distribution and its Applications." Communications in Statistics - Theory and Methods, 31, 497-512.

Gradshteyn IS, Ryzhik IM (2000). Table of Integrals, Series, and Products. Academic Press, New York.

Hosking JRM (1990). "L-moments: Analysis and Estimation of Distributions Using Linear Combinations of Order Statistics." Journal of the Royal Statistical Society (Series B), 52, 105-124.

Jones MC (2004). "Family of Distributions Arising from Distribution of Order Statistics." Test, 13, $1-43$.

Lee C, Famoye F, Olumolade O (2007). "Beta-Weibull Distribution: Some Properties and Applications to Censored Data." Journal of Modern Applied Statistical Methods, 6, 173-186.

Mudholkar GS, Srivastava DK (1993). "Exponentiated Weibull Family for Analyzing Bathtub Failurerate Data." IEEE Transactions on Reliability, 42, 299-302.

Nelson W (1982). Applied Life Data Analysis. John Wiley and Sons, New York.

Pal M, Masoom Ali M, Woo J (2006). “On the Exponentiated Weibull Distribution.” Statistica, 66, 139-147.

Prudnikov AP, Brychkov YA, Marichev OI (1986). Integrals and Series, volume 1. Gordon and Breach Science Publishers, Amsterdam.

Quesenberry CP, Kent J (1982). "Selecting Among Probability Distributions used in Reliability." Technometrics, 24, 59-65.

Sarhan AM, Zaindin M (2009). "Modified Weibull Distribution.” Applied Sciences, 11, 123-136.

Silva GO, Ortega EMM, Cordeiro GM (2010). “The Beta Modified Weibull Distribution.” Lifetime Data Analysis, 16, 409-430.

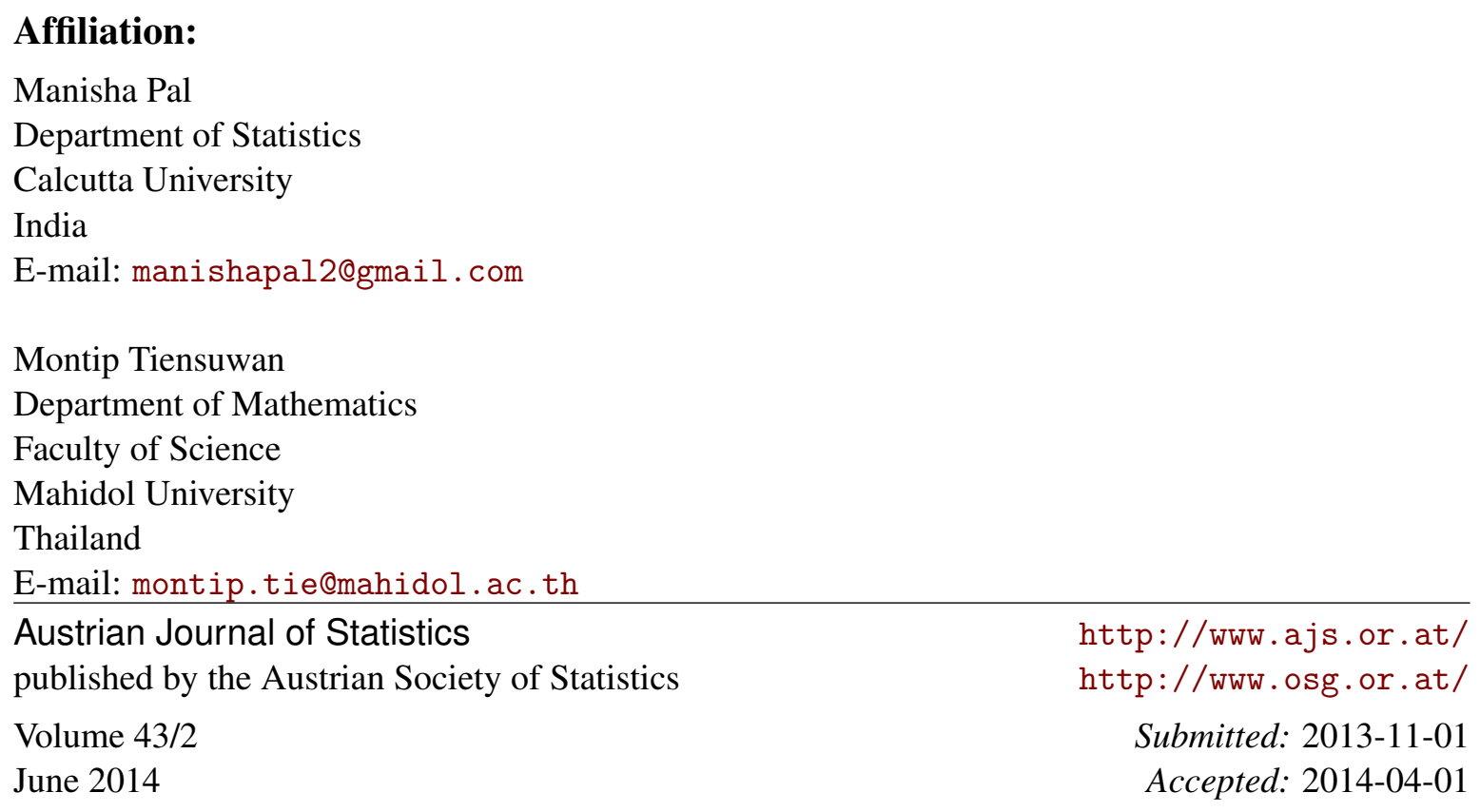

